\title{
ON A CLASS OF RANDOM VARIABLES
}

FRANK SPITZER

1. Introduction. The purpose of this note is to point out a connection between the theory of probability and the mean value theorems of classical analysis. This connection will be exploited to study a class of random variables which give rise to interesting mean value theorems for analytic functions of a complex variable. As an illustration, consider the mean value theorem

$$
\frac{1}{2 \pi} \int_{0}^{2 \pi} f\left(r e^{i \theta}\right) d \theta=f(0)
$$

where $f\left(r e^{i \theta}\right)=f(z)$ is any analytic function regular in a circle of radius $R>r$ about the origin. We can define a particular complex valued random variable $Z$ in the following way: $|Z|=r$ with probability one, and $\arg Z$ is uniformly distributed on the interval $[-\pi, \pi]$. We shall denote by $E$ the linear operator which "takes the expected value" of $Z$ and of measurable functions of $Z$ with respect to the probability measure we have defined. Then $E[Z]=0$, and the first member of (1.1) is clearly $E[f(Z)]$, so that (1.1) may be written in the form

$$
E[f(Z)]=f[E(Z)] \text {. }
$$

Equation (1.2) will be the point of departure for this discussion. We shall study complex valued random variables $Z$ with the property that (1.2) holds for all suitably restricted analytic functions $f(z)$.

2. Complex valued regular random variables. To anticipate difficulties arising from problems of measurability we shall restrict the classes of complex valued random variables ${ }^{1} Z$ and of analytic functions $f(z)$ under consideration. $f(z)$ will be a member of the class of polynomials in $z$, while $Z$ will be assumed to possess finite absolute moments of all orders. Then both members of equation (1.2) are seen to be finite, although not necessarily equal. We shall now use (1.2) to define a class of random variables.

Definition. A complex valued random variable $Z$ is said to be a regular random variable (r.r.v.), if

Presented to the Society, September 2, 1954 ; received by the editors July $14,1954$.

1 A complex valued random variable $Z$ is a pair of real valued random variables $X$ and $Y$, in the sense that $Z$ takes the values $X+i Y . X$ and $Y$ are not assumed independent, so that every probability measure in the plane determines a complex valued random variable. 


$$
E\left[|Z|^{n}\right]<\infty, \quad n=1,2, \cdots,
$$

and if

$$
E[f(Z)]=f[E(Z)]
$$

for every polynomial $f(z)$.

Regular random variables, as defined, have the following basic properties:

THEOREM 1. (a) If $Z$ is a r.r.v., and if $f(z)$ is a polynomial, then $f(Z)$ is again a r.r.v.

(b) If $Z_{1}$ and $Z_{2}$ are independent (in the sense of probability) r.r.v.'s, then $Z_{1}+Z_{2}$ is again a r.r.v.

Proof. (a) Since $E\left[|Z|^{n}\right]<\infty$ for all $n$ and since $f(z)$ is a polynomial, we have $E\left[|f(Z)|^{n}\right]<\infty$ for all $n$. It remains to show that for every polynomial we have $E[g\{f(Z)\}]=g[E\{f(Z)\}]$. Simplifying the notation, we have $\operatorname{Eg} f(Z)=g f E(Z)$ because $g f$ is a polynomial, and $g f E(Z)=g E f(Z)$ because $Z$ is a r.r.v.

(b) $E\left[\left|Z_{1}+Z_{2}\right|^{n}\right] \leqq E\left[\left(\left|Z_{1}\right|+\left|Z_{2}\right|\right)^{n}\right]<\infty$. Since $Z_{1}$ and $Z_{2}$ are independent r.r.v.'s with expectation operators $E_{1}$ and $E_{2}$, we have for an arbitrary polynomial $f(z)$

$$
\begin{aligned}
E f\left(Z_{1}+Z_{2}\right) & =E_{2} E_{1} f\left(Z_{1}+Z_{2}\right)=E_{2} f\left[E_{1}\left(Z_{1}\right)+Z_{2}\right] \\
& =f\left[E_{1}\left(Z_{1}\right)+E_{2}\left(Z_{2}\right)\right]=f\left[E\left(Z_{1}+Z_{2}\right)\right] .
\end{aligned}
$$

That completes the proof.

Next we consider the real and imaginary parts $X$ and $Y$ of a r.r.v. $Z=X+i Y$. Without loss of generality we may assume that $E[Z]=0$, since by Theorem 1 the translate $Z-E[Z]$ of a r.r.v. is again a r.r.v. Theorem 1(a) applied to the polynomial $f(z)=z^{2}$ at once yields the

CoROLlaRY. The real and imaginary parts $X$ and $Y$ of a r.r.v. with mean zero form a pair of real valued orthogonal random variables with the same variance, i.e.

$$
E(X)=E(Y)=E(X Y)=E\left(X^{2}\right)-E\left(Y^{2}\right)=0 .
$$

It is clear from the example given in the introduction (1.1) that $X$ and $Y$, although orthogonal, will not in general be independent. Moreover, not every pair of orthogonal random variables can constitute the real and imaginary parts of a r.r.v. This motivates the

Definition. The couple of real valued random variables $X$ and $Y$ is called a couple of conjugate random variables if and only if $Z=X+i Y$ is a r.r.v.

A couple of conjugate random variables is therefore determined by 
a probability measure in the plane, just as is a r.r.v. In order to further characterize such measures, let $\Phi(\alpha, \beta)$ denote the symbolic power series

$$
\Phi(\alpha, \beta)=\sum_{n=0}^{\infty} \frac{(i)^{n}}{n !} E\left[(\alpha X+\beta Y)^{n}\right],
$$

where $X$ and $Y$ are conjugate random variables with mean zero. The variables $\alpha$ and $\beta$ are taken to be complex valued. We are not assuming that the bivariate characteristic function

$$
\phi(\alpha, \beta)=E\left[e^{i \alpha X+i \beta Y}\right] \quad(\alpha, \beta \text { real })
$$

exists for complex values of $\alpha$ and $\beta$. Therefore $\Phi(\alpha, \beta)$ must, in what follows, be interpreted as a symbolic power series in $\alpha$ and $\beta$. Only if there exists a neighborhood $N$ of the origin in the complex plane such that the series $\Phi(\alpha, \beta)$ converges for all $\alpha$ and $\beta$ in $N$ can it be asserted that this series represents the characteristic function, i.e. that $\phi(\alpha, \beta) \equiv \Phi(\alpha, \beta)$ for all $\alpha$ and $\beta$ in $N$. Given $\phi(\alpha, \beta)$ for real $\alpha$ and $\beta$, the coefficients of the power series $\Phi(\alpha, \beta)$ are, however, uniquely determined. They are known to equal the coefficients of the MacLaurin expansion of $\phi(\alpha, \beta)$, because the moments of $X$ and $Y$ are finite. On the other hand, given $\Phi(\alpha, \beta), \phi(\alpha, \beta)$ need not be uniquely determined. The following theorem shows how r.r.v.'s can be characterized in terms of their $\Phi(\alpha, \beta)$.

TheOREM 2. Let $Z=X+i Y$ be a complex valued random variable with mean zero, all of whose absolute moments exist. Then $Z$ is a r.r.v. if and only if

$$
\Phi(\alpha, i \alpha) \equiv 1
$$

for all complex $\alpha$.

Proof. Because $E\left[|Z|^{n}\right]<\infty$ for all $n, \Phi(\alpha, \beta)$ is well defined by (2.1). Suppose that (2.2) holds. Then, after substituting $\beta=i \alpha$ into (2.1) the coefficients of $\alpha^{n}$ must vanish for $n \geqq 1$, i.e. we have $E[(X$ $\left.+i Y)^{n}\right]=0$ for $n \geqq 1$. If $f(z)$ is a polynomial, it follows that $\operatorname{Ef}(Z)$ $=f(0)=f E(Z)$, so that $Z$ is a r.r.v. Conversely, if $Z$ is a r.r.v. with $E[Z]=0$, then it satisfies (1.2) for $f(z)=z^{n}$. Hence we have $E\left[Z^{n}\right]=0$ for $n \geqq 1$, so that $\Phi(\alpha, i \alpha) \equiv 1$, by $(2.1)$.

It is well known that not only the random variable of (1.1), but all other radially symmetric complex valued random variables satisfy the mean value theorem (1.2). That this is so can be verified from Theorem 2. By radial symmetry about the origin we mean that the probability measure of $Z$ is invariant under all rotations about the 
origin, i.e. that $e^{i \theta} Z$ has the same probability measure as $Z$ for all real values of $\theta$. It is known [1] that a radially symmetric random variable $Z$ has a characteristic function of the form

$$
\phi(\alpha, \beta)=E\left[e^{i \alpha X+i \beta Y}\right]=\int_{0}^{\infty} J_{0}\left(R\left(\alpha^{2}+\beta^{2}\right)^{1 / 2}\right) d F(R)
$$

for real $\alpha$ and $\beta . J_{0}(x)$ is the Bessel function of order zero, and $F(x)$ is a probability distribution on $[0, \infty)$. If, in addition, all the moments of $F(x)$ exist, then (2.3) determines uniquely the coefficients of $\Phi(\alpha, \beta)$ as defined in (2.1). It follows from (2.3) that $\Phi(\alpha, i \alpha) \equiv 1$, i.e. that $Z$ is a r.r.v.

A well-known radially symmetric complex valued random variable is $Z=X+i Y$, where $X$ and $Y$ are independent, normal, and have the same variance. Clearly $Z$ is a r.r.v., in fact

$$
\begin{aligned}
\frac{1}{2 \pi \sigma^{2}} \int_{-\infty}^{\infty} \int_{-\infty}^{\infty} f(x+i y) \exp & {\left[-\frac{(x-a)^{2}}{2 \sigma^{2}}\right] } \\
\cdot \exp & {\left[-\frac{(y-b)^{2}}{2 \sigma^{2}}\right] d x d y \equiv f(a+i b) }
\end{aligned}
$$

for every entire function $f(z)$ of order less than two. $Z$, as defined here, is known to be the only radially symmetric complex valued random variable whose real and imaginary parts are independent random variables [2]. We shall now investigate whether it is the only r.r.v. with independent real and imaginary parts. Of course the two dimensional Dirac $\delta$-function is such a r.r.v. (a unit mass concentrated at a point), but we shall show that there are still other probability distributions $F(x)$, such that

$$
\int_{-\infty}^{\infty} \int_{-\infty}^{\infty} f(x+i y) d_{x} F(x-a) d_{y} F(y-b) \equiv f(a+i b)
$$

for every polynomial $f(z)$, i.e. that there are other r.r.v.'s with independent identically distributed real and imaginary parts. For an example, see equations (4.1) to (4.3). Equation (2.5) suggests that such r.r.v.'s may, in a very natural way, be regarded as a generalization of the Dirac $\delta$-function, and hence as a generalization of complex numbers.

\section{Regular random variables with independent real and imaginary} parts. We shall now formulate the main theorem concerning such r.r.v.'s. Let $X$ and $Y$ be independent real-valued random variables with finite moments. Let $\Phi_{x}(\alpha)$ and $\Phi_{y}(\beta)$ be the symbolic power series 


$$
\Phi_{x}(\alpha)=\sum_{n=0}^{\infty} \frac{(i \alpha)^{n}}{n !} E\left(X^{n}\right), \quad \Phi_{y}(\beta)=\sum_{n=0}^{\infty} \frac{(i \beta)^{n}}{n !} E\left(Y^{n}\right),
$$

for complex $\alpha$ and $\beta$.

TheOREM 3. $X$ and $Y$ are conjugate, with mean zero, i.e. $Z=X+i Y$ is a r.r.v. with mean zero, if and only if

$$
\Phi_{x}(\alpha) \Phi_{y}(i \alpha) \equiv 1
$$

Further, (3.1) holds if and only if

$$
\begin{array}{r}
E\left[X^{2 n+1}\right]=E\left[Y^{2 n+1}\right]=\sum_{k=0}^{2 n}(-1)^{k}\left(\begin{array}{l}
2 n \\
2 k
\end{array}\right) E\left[X^{2 k}\right] E\left[Y^{2 n-2 k}\right]=0 \\
n=1,2, \cdots .
\end{array}
$$

Proof. Term by term multiplication of the power series in (3.1) yields

$$
\sum_{n=0}^{\infty}(i \alpha)^{n} \sum_{k=0}^{n} \frac{(i)^{n-k}}{(n-k) ! k !} E\left(X^{k}\right) E\left(Y^{n-k}\right) \equiv 1,
$$

or $E\left[(X+i Y)^{n}\right]=0$ for $n \geqq 1$. This proves the first part of the theorem. Also (3.2) clearly implies that $E\left[Z^{n}\right]=0$ for $n \geqq 1$, i.e. that $Z$ is a r.r.v. Finally, if we assume that $E\left[Z^{k}\right]=0$ for $k \geqq 1$, the second half of (3.2) follows by choosing $k$ to be even. The first half of (3.2) follows from $E\left[Z^{k}\right]=0$ by induction. For assume that $E\left[X^{2 k+1}\right]=E\left[Y^{2 k+1}\right]=0$ for $k=0,1, \cdots, n-1$. Then $E\left[(X+i Y)^{2 n+1}\right]=0$ implies that

$$
E\left[X^{2 n+1}\right]+(i)^{2 n+1} E\left[Y^{2 n+1}\right]=0 .
$$

Hence all odd moments vanish, by induction. This completes the proof.

It remains to exhibit independent conjugate random variables which are neither normal, nor constants. It is easily verified that $\phi_{x}(\lambda)=\cos \lambda$ and $\phi_{y}(\lambda)=[\cosh \lambda]^{-1}$ are both characteristic functions of real-valued random variables, which we may call $X$ and $Y$. Since both characteristic functions are regular in $\lambda$ for $|\lambda|<\pi / 2$, we have for complex $\lambda$

$$
\Phi_{x}(\lambda) \Phi_{y}(i \lambda) \equiv 1,
$$

and it follows from Theorem 3 that, if $X$ and $Y$ are taken to be independent, $Z=X+i Y$ is a r.r.v. Incidentally,

$$
Z=\tanh (\pi / 4) W
$$

where $W$ is the r.r.v. which is defined by saying that $|W|=1$ with 
probability one, and that $\arg W$ is uniformly distributed on $[-\pi, \pi]$. We note that $Z$ is unbounded in the $y$-direction. Indeed, there are no bounded r.r.v.'s with independent real and imaginary parts, if we except the trivial case of a point mass. For suppose $Z=X+i Y$ were a r.r.v., bounded with probability one. It is well-known that the characteristic functions $\phi_{x}(\lambda)$ and $\phi_{y}(\lambda)$ will then have to be entire functions of order one. But Theorem 3 implies that

$$
\phi_{x}(\lambda) \phi_{y}(i \lambda) \equiv 1,
$$

if we assume that $Z$ has been translated to have mean zero. Since $\phi_{y}(\lambda)$ is entire, $\phi_{x}(\lambda)$ has no zeros. An entire function of $\lambda$ of order one which has no zeros is of the form $\exp (A \lambda+B)$. Hence $Z$ must be the unit mass at the origin, under the assumption of boundedness and mean zero.

4. Self-conjugate random variables. We shall briefly consider a class of real-valued random variables which arise in the following way.

Definition. A real-valued random variable is said to be self-conjugate, if $Z=X+i Y$ is a r.r.v. with mean zero when $Y$ is independent of $X$ and has the same distribution as $X$.

First an example is constructed from the example in the previous section. We form

$$
\phi(\lambda)=\phi_{x}(\lambda) \phi_{y}(\lambda)=\cos \lambda / \cosh \lambda .
$$

It is easily verified that $\phi(\lambda)$ is the characteristic function of a realvalued random variable $X$ which has the probability density

$$
\begin{aligned}
g(x) & =\frac{1}{2 \pi} \int_{-\infty}^{\infty} e^{-i \lambda x} \frac{\cos \lambda}{\cosh \lambda} d \lambda \\
& =\frac{1}{4}\left[\frac{1}{\cosh (\pi(x-1) / 2)}+\frac{1}{\cosh (\pi(x+1) / 2)}\right] .
\end{aligned}
$$

Since $\phi(\lambda) \phi(i \lambda)=1, X$ is a self-conjugate real-valued random variable. If $Y$ is taken to be distributed as $X$ and to be independent of $X$, then $Z=X+i Y$ is a r.r.v. The mean value theorem for analytic functions corresponding to equation (1.2), when applied to the present r.r.v. $Z$, becomes:

$$
\int_{-\infty}^{\infty} \int_{-\infty}^{\infty} f(x+i y) g(x-a) g(y-b) d x d y \equiv f(a+i b),
$$

with $g(x)$ given by (4.2), and (4.3) holds for every polynomial, and in fact for every entire function $f(z)$ of order less than one. 
According to its definition and Theorem 3, a random variable $X$ is self-conjugate if and only if

$$
\Phi(\alpha) \Phi(i \alpha) \equiv 1, \quad \Phi(\alpha) \equiv \Phi(-\alpha),
$$

where $\Phi(\alpha)$ is the symbolic power series

$$
\Phi(\alpha)=\sum_{n=0}^{\infty} \frac{(i \alpha)^{n}}{n !} E\left(X^{n}\right) .
$$

Regardless of whether $\Phi(\alpha)$ converges or not we may form the symbolic power series $\log \Phi(\alpha)$, which has the following definition:

$$
\log \Phi(\alpha)=\log \left[1+\sum_{n=1}^{\infty} \frac{(i \alpha)^{n}}{n !} E\left(X^{n}\right)\right],
$$

where the right side is to be expanded in powers of $\alpha$ by using the power series for $\log (1+z)$. If $X$ is self-conjugate, it follows from (4.4) that

$$
\log \Phi(\alpha)+\log \Phi(i \alpha) \equiv 0, \quad \log \Phi(\alpha) \equiv \log \Phi(-\alpha) .
$$

If a random variable $X$ has finite moments the coefficients $a_{n}$ in

$$
\log \Phi(\alpha)=\sum_{n=1}^{\infty} a_{n} \alpha^{n}
$$

are uniquely determined by the moments. The $a_{n}$, apart from normalizing constants, are known as the semi-invariants of $X$. The logarithm of the characteristic function $\phi(\lambda)$ of a random variable with finite moments has a MacLaurin expansion. Hence the coefficients of log $\Phi(\alpha)$ are given by

$$
a_{n}=\frac{1}{n !} \frac{d^{n}}{d \lambda^{n}} \log \phi(0)
$$

Combining equations (4.5), (4.6), and (4.7), it is evident that we have proved

THEOREM 4. Let $\phi(\lambda)$ be a characteristic function. Then it is the characteristic function of a self-conjugate random variable if and only if

$$
\left(d^{n} / d \lambda^{n}\right) \log _{\AA}^{*} \phi(0)_{-}^{*}=0,
$$

when $n$ is odd or a multiple of four.

This theorem is far from final, in that it answers an easy question but raises a difficult one: which functions $\phi(\lambda)$, satisfying (4.8), are 
characteristic functions? This is an open problem, but the following remarks have bearing on it.

REMARK 1. If

$$
\phi(\lambda)=\prod_{\nu=1}^{\infty} \frac{1-\lambda^{2} / \delta_{\nu}^{2}}{1+\lambda^{2} / \delta_{\nu}^{2}} e^{-k \lambda^{2}}, \quad \sum_{\nu=1}^{\infty} \frac{1}{\delta_{\nu}^{2}}<\infty, \quad \delta_{\nu}^{2}>0,
$$

then $\phi(\lambda)$ is the characteristic function of a self-conjugate random variable for large enough positive values of $k$. (The proof is based on the fact that

$$
\frac{1-\lambda^{2}}{1+\lambda^{2}} \exp \left[-\frac{A}{2} \lambda^{2}\right]
$$

is a characteristic function for $A \geqq 1$. The above infinite product converges to a characteristic function which evidently satisfies (4.8).)

REMARK 2. The following fact is a consequence of Remark 1 , and of the work of Pólya [3] on the zeros of entire functions. Let $f(x)$ be positive, integrable and nondecreasing on the unit interval. Let

$$
\Psi(\lambda)=\int_{0}^{1} f(x) \cos \lambda x d x .
$$

Then

$$
\phi(\lambda)=\Psi(\lambda) / \Psi(i \lambda)
$$

is the characteristic function of a self-conjugate random variable.

REMARK 3. Theorem 4 suggests the question whether there exist characteristic functions all of whose semi-invariants vanish from a certain point on. The answer is negative, with the obvious exception of the normal random variables. In other words, it is known that, if a polynomial $P(\lambda)$ is the logarithm of a characteristic function, then $P(\lambda)$ is at most of degree two [4].

REMARK 4. The self-conjugate laws enjoy the following type of group property which is an immediate consequence of Theorem 4 . Let $\phi_{1}(\lambda), \phi_{2}(\lambda)$, and $\phi_{3}(\lambda)$ be characteristic functions, and suppose that $\phi_{1}(\lambda) \phi_{2}(\lambda)=\phi_{3}(\lambda)$. If one assumes that any two of these are selfconjugate, then it follows that the third one is also self-conjugate.

It is known that this group property is not shared by the class of infinitely divisible laws. The normal random variables are evidently both infinitely divisible and self-conjugate. However, they are the only ones with this property. This is easily verified by applying (4.8) to the Lévy-Khintchine representation for the logarithm of an infinitely divisible characteristic function. 
5. A generalization to $n$ dimensions. We shall now define regular random variables in $n$ dimensions. The defining relation (1.2) for the case of two dimensions could have been written

$$
E\left[u\left(X_{1}, X_{2}\right)\right]=u\left[E\left(X_{1}\right), E\left(X_{2}\right)\right],
$$

where the couple $\left\{X_{1}, X_{2}\right\}$ is a two-dimensional vector random variable, and where $u\left(x_{1}, x_{2}\right)$ is a harmonic function of the real variables $x_{1}, x_{2}$. In $n$ dimensions we shall accordingly deal with vector random variables $X=\left\{X_{1}, X_{2}, \cdots, X_{n}\right\}$, having as their components $n$ realvalued random variables which may or may not be independent. $u(x)=u\left(x_{1}, x_{2}, \cdots, x_{n}\right)$ will be restricted to be a harmonic polynomial, i.e. a polynomial in $x_{1}, x_{2}, \cdots, x_{n}$, which satisfies Laplace's equation in $n$ dimensions. The coefficients of $u(x)$ may be complex. We shall write $u[E(X)]$ for $u\left[E\left(X_{1}\right), \cdots, E\left(X_{n}\right)\right]$.

Definition. $X$ is said to be an $n$-dimensional regular random vari$a b l e$, if all the moments of its components exist, and if

$$
E[u(X)]=u[E(X)]
$$

for every harmonic polynomial $u(\mathbf{x})$.

Our previous definition of a r.r.v. in two dimensions is clearly in agreement with the above definition. In the case of one dimension $X$ reduces to an ordinary real-valued random variable. $u(x)$ reduces to a linear function $a x+b$. Since $E$ is a linear operator, every real-valued random variable with finite moments is a one dimensional r.r.v.

To see that there exist r.r.v.'s in three and higher dimensions, we turn again to the radially symmetric case. Suppose that $E(X)=0$ and that the probability measure of $X$ is left invariant by rotations about the origin, and that in addition the moments of $X$, and hence of $\left(X_{1}^{2}+\cdots+X_{n}^{2}\right)^{1 / 2}$, are finite. The probability measure of $X$ may be written $d F d \mu$, where the radial part $F$ is a probability distribution on the positive real numbers with finite moments, and where $\mu$ is the uniform distribution over an $(n-1)$-sphere $S$ about the origin. Since $u(\mathrm{x})$ is measurable with regard to $d F d \mu$ we have

$$
E[u(\boldsymbol{X})]=\lim _{R \rightarrow \infty} \int_{0}^{R} d F(\rho) \int_{S} u\left(\rho, \theta_{1}, \cdots, \theta_{n-1}\right) d \mu\left(\theta_{1}, \cdots, \theta_{n-1}\right) .
$$

By the mean value theorem for harmonic functions

$$
\int_{S} u d \mu \equiv u(0)
$$

for every value of $\rho$, so that $E[u(X)]=u(0)=u[E(X)]$. 
The above discussion proves that $X$ is a r.r.v. if its components $X_{i}(i=1,2, \cdots, n)$ are mutually independent normal random variables with the same variance. The following theorem will show that in three and higher dimensions there are no other r.r.v.'s with independent components.

THEOREM 5. Let $X$ be a regular random variable in $n$ dimensions $(n \geqq 3)$, whose components are independent. Then the components of $X$ are all normally distributed with the same variance.

Proof. It will suffice to prove the theorem for the case $n=3$. For suppose that $X_{\alpha}, X_{\beta}, X_{\gamma}$ are three arbitrary components of an $n$-dimensional r.r.v. $X$. The definition of $X$ requires (5.1) to hold for any harmonic polynomial in $X_{\alpha}, X_{\beta}, X_{\gamma}$, which were arbitrarily selected. Hence all the components of $X$ will have been shown to be normal if we prove the theorem for $n=3$.

There is obviously no loss of generality in assuming that $E(X)=0$. We further note that the components of $X=\left\{X_{1}, X_{2}, X_{3}\right\}$ must have the same variance since $X_{1}+i X_{2}$ and $X_{1}+i X_{3}$ have to be two-dimensional r.r.v.'s. For the same reason the odd moments of the $X_{i}$ must vanish.

Now assume the theorem to be false. Then we may define the threedimensional random variables $X^{(1)}=\left\{X_{1}, X_{2}, X_{3}\right\}, X^{(2)}=\left\{X_{2}, X_{3}, X_{1}\right\}$, and $X^{(3)}=\left\{X_{3}, X_{1}, X_{2}\right\}$. We may assume (the theorem being assumed false), that $X^{(1)}$ is a r.r.v. with independent components, and that at least one of these components is not normally distributed. It follows that $\boldsymbol{X}^{(2)}$ and $\boldsymbol{X}^{(3)}$ are also r.r.v.'s. Now $\boldsymbol{X}^{(1)}, \boldsymbol{X}^{(2)}, \boldsymbol{X}^{(3)}$ are taken to be independent, and we form $Y=X^{(1)}+X^{(2)}+X^{(8)}$. The argument used to prove Theorem 1 shows that $Y$ is a r.r.v. It follows from the definition of $Y$ that its components are independent and identically distributed. Each component $Y_{i}=X_{1}+X_{2}+X_{3}(i=1,2,3)$ is the sum of three independent random variables which are not all normal. By a well-known theorem due to Cramér [5] the distribution of the $Y_{i}(i=1,2,3)$ can therefore not be normal.

So far it has been shown that, if the theorem is false, then there exists a r.r.v. $\boldsymbol{Y}$ with independent identically distributed, non-normal components. The proof will now be completed by assuming that $X=\left\{X_{1}, X_{2}, X_{3}\right\}$ is a r.r.v. with independent, identically distributed components, and by showing that the $X_{i}$ must be normal. As mentioned, we shall also assume that $E\left(X_{i}\right)=0$ and we may even postulate that $X$ has been normalized so that $E\left(X_{i}^{2}\right)=1$. Let

$$
u_{n}(\mathbf{x})=\left[\frac{x_{1}}{2^{1 / 2}}+\frac{x_{2}}{2^{1 / 2}}+i x_{3}\right]^{n} .
$$


It can be checked that the $u_{n}(\mathrm{x})$ are harmonic polynomials. By equation (5.1) we have

$$
E\left[u_{n}(X)\right]=0, \quad n=1,2,3, \cdots .
$$

It is easily verified that (5.2) holds for $n=2$ and for all odd $n$ (since odd moments of the $X_{i}$ are zero). The second moment of the $X_{i}$ having been fixed in advance, we shall now show that equation (5.2) uniquely determines the other moments $E\left[X_{i}^{2 k}\right]$. For assume that $E\left[X_{i}^{k}\right]$ has been uniquely determined by our assumptions for $k=1$, $2, \cdots, 2 p-1$. Then (5.2) for the case $n=2 p$ can be written in the form

$$
A_{p}+E\left[2^{-p} X_{1}^{2 p}+2^{-p} X_{2}^{2 p}+(-1)^{p} X_{3}^{2 p}\right]=0,
$$

where $A_{p}$ is a function of the known moments of order up to and including $2 p-1$. Equation (5.3) now determines $E\left[X_{1}^{2 p}\right]$ uniquely, and the next moment, being an odd moment, is zero. By induction it follows that all the moments of the $X_{i}(i=1,2,3)$ are uniquely determined by our assumptions.

Not all random variables are uniquely determined by their moments, but the normal ones are. We have seen that, because of its radial symmetry, $X=\left\{X_{1}, X_{2}, X_{3}\right\}$ is a r.r.v. which satisfies our assumption if each $X_{i}$ is normal with mean zero and variance one. Hence its moments are those determined recursively by equation (5.3), and the proof is complete.

Recapitulating, Theorem 5 states that if

$$
\begin{aligned}
\int_{-\infty}^{\infty} \cdots \int_{-\infty}^{\infty} u\left(x_{1}, x_{2}, \cdots, x_{n}\right) & \prod_{i=1}^{n} d_{x_{i}} F_{i}\left(x_{i}-a_{i}\right) \\
& \equiv u\left(a_{1}, a_{2}, \cdots, a_{n}\right), \quad n \geqq 3,
\end{aligned}
$$

for a class of harmonic functions $u(x)$ containing the harmonic polynomials, then the distribution functions $F_{i}(x)$ are all of the form

$$
F_{i}(x)=\frac{1}{(2 \pi)^{1 / 2}} \int_{-\infty}^{x / t^{1 / 2}} \exp \left[-\frac{1}{2} s^{2}\right] d s, \quad i=1,2, \cdots, n,
$$

where $t>0$ is their common variance. We shall now show that the converse is also true, indeed for $n \geqq 1$, in the following sense: If (5.4) holds, with the $F_{i}(x)$ given by (5.5) for a function $u(\mathrm{x})$, then $u(\mathrm{x})$ must be a harmonic function. This is reexpressed in

THEOREM 6. Let $X=\left\{X_{1}, \cdots, X_{n}\right\}$ be the vector random variable whose components are mutually independent and normal with mean zero and variance one. Let $u(\mathrm{x})$ be a complex valued function of $\mathrm{x}=\left\{x_{1}, \cdots\right.$, 
$\left.x_{n}\right\}$ such that

(5.6) $E\left[\left|u\left(t^{1 / 2} X+a\right)\right|\right]<\infty$ for all $t \geqq 0$, and all $\mathbf{a}=\left\{a_{1}, \cdots, a_{n}\right\}$, (5.7) $E\left[u\left(t^{1 / 2} X+a\right)\right] \equiv u(a)$ for all $t \geqq 0$, and all $a=\left\{a_{1}, \cdots, a_{n}\right\}$. Then $u(x)$ is an entire harmonic function.

Proof. The assumption (5.7) of the theorem is equivalent to (5.4), with $F_{i}(x)(i=1,2, \cdots, n)$ given by $(5.5)$. Let $\nabla_{a}^{2}$ be the Laplacian operator

$$
\nabla_{a}^{2}=\frac{\partial^{2}}{\partial a_{1}^{2}}+\cdots+\frac{\partial^{2}}{\partial a_{n}^{2}} .
$$

By assumption (5.6) of the theorem we may apply $\nabla_{a}^{2}$ to the left side of (5.4) and justify an interchange of the order of differentiation and integration. We obtain

$$
\int_{-\infty}^{\infty} \cdots \int_{-\infty}^{\infty} u(\mathrm{x}) \nabla_{a}^{2} \prod_{i=1}^{n} d_{x_{i}} F_{i}\left(x_{i}-a_{i}\right) \equiv \nabla_{a}^{2} u(\mathrm{a})
$$

But by (5.5)

$$
\nabla_{a}^{2} \prod_{i=1}^{n} F_{i}\left(x_{i}-a_{i}\right)=2 \frac{\partial}{\partial t} \prod_{i=1}^{n} F_{i}\left(x_{i}-a_{i}\right)
$$

Hence

$$
2 \frac{\partial}{\partial t} \int_{-\infty}^{\infty} \cdots \int_{-\infty}^{\infty} u(\mathrm{x}) \prod_{i=1}^{n} d_{x_{i}} F_{i}\left(x_{i}-a_{i}\right) \equiv \nabla_{a}^{2} u(\mathrm{a}) .
$$

The left-hand side vanishes by our assumption (5.7), so that

$$
\nabla_{\mathrm{a}}^{2} u(a)=0 \text { for all } a=\left\{a_{1}, \cdots, a_{n}\right\} .
$$

This completes the proof.

\section{BIBLIOGRAPHY}

1. S. Bochner and K. Chandrasekharan, Fourier transforms, Princeton University Press, 1949.

2. M. Kac, $A$ characterization of the normal distribution, Amer. J. Math. vol. 61 (1939) pp. 726-728.

3. G. Polya, Über die Nullstellen gewisser ganzer Funktionen, Math. Zeit. vol. 41 (1936) pp. 352-383.

4. J. Marcinkiewicz, Sur une propriete de la loi de gauss, Math. Zeit. vol. 44 (1938) pp. 612-618.

5. H. Cramér, Über eine Eigenschaft der normalen Verteilungsfunktion, Math. Zeit. vol. 41 (1936) pp. 405-414.

California Institute of Technology 